\title{
Molecular Mechanisms and Therapeutics for SCA17
}

\author{
Qiong Liu ${ }^{1,2} \cdot$ Yongcheng $\mathrm{Pan}^{1,2} \cdot$ Xiao-Jiang $\mathrm{Li}^{2} \cdot$ Shihua $\mathrm{Li}^{2}$
}

Published online: 17 July 2019

(C) The American Society for Experimental NeuroTherapeutics, Inc. 2019

\begin{abstract}
Spinocerebellar ataxia type 17 (SCA17) is caused by polyglutamine (polyQ) expansion in the TATA box-binding protein (TBP), which functions as a general transcription factor. Like other polyQ expansion-mediated diseases, SCA17 is characterized by lateonset and selective neurodegeneration, despite the disease protein being ubiquitously expressed in the body. To date, the pathogenesis of polyQ diseases is not fully understood, and there are no effective treatments for these devastating disorders. The well-characterized function of TBP and typical neurodegeneration in SCA17 give us opportunities to understand how polyQ expansion causes selective neurodegeneration and to develop effective therapeutics. In this review, we discuss the molecular mechanisms behind SCA17, focusing on transcriptional dysregulation as its major cause. Mounting evidence suggests that reversing transcriptional alterations induced by mutant TBP and reducing the expression of mutant TBP are promising strategies to treat SCA17.
\end{abstract}

Key Words Polyglutamine $\cdot$ SCA17 $\cdot$ TBP $\cdot$ transcription $\cdot$ neurodegeneration $\cdot$ muscle atrophy

\section{Introduction}

Polyglutamine (polyQ) diseases are a group of neurodegenerative disorders caused by CAG trinucleotide repeat expansion that encodes an expanded polyQ tract in the disease proteins [1]. Currently, we know of a total of nine polyQ disorders: six spinocerebellar ataxias (SCA), types 1, 2, 3, 6, 7, and 17; Huntington's disease (HD); dentatorubral-pallidoluysian atrophy (DRPLA); and spinobulbar muscular atrophy [1]. Among these diseases, SCA17 is the latest addition to the family. In 1999, Koide et al. first reported a 14-year-old Japanese patient with unique neurologic symptoms associated with de novo $\mathrm{CAG} / \mathrm{CAA}$ repeat expansion in the gene encoding the TATA box-binding protein (TBP) [2]. In 2001, this abnormal CAG/CAA expansion in the TBP gene was verified in four Japanese pedigrees, leading

Xiao-Jiang Li

xjli33@jnu.edu.cn

1 Key Laboratory of Hunan Province in Neurodegenerative Disorders, Xiangya Hospital, Central South University, Changsha 410008, Hunan, China

2 Guangdong-Hongkong-Macau Institute of CNS Regeneration, Ministry of Education CNS Regeneration Collaborative Joint Laboratory, Jinan University, Guangzhou, China to a neurological disorder inherited in an autosomal dominant manner; this was classified as SCA17, an additional polyQ diseases [3].

Although most TBP mutations in SCA17 patients are inherited as an autosomal dominant trait, de novo mutations have also been reported [2, 4-6]. Repeat expansion in SCA17 is different from the pure expanded CAG tract, as it is interrupted by CAA repeats that can stabilize the repeats for transmission between generations; however, there are a few reports of intergenerational instability in German and Italian families [7, 8]. Previous studies have shown that the wild-type alleles of TBP have 25 to $42 \mathrm{CAG} / \mathrm{CAA}$ repeats, whereas most SCA17 alleles range between 46 and $55 \mathrm{CAG} / \mathrm{CAA}$ repeats and are responsible for the classic adult-onset symptoms, including ataxia, dystonia, parkinsonism, choreic movements, dementia, psychiatric abnormalities, and seizures [2, 3, $9,10]$. Brain magnetic resonance imaging (MRI) usually shows marked cerebellar atrophy and degeneration, accompanied by moderate and diffuse cortical and brainstem atrophy $[3,10]$. Repeats exceeding $62 \mathrm{CAG} / \mathrm{CAA}$ typically result in juvenile-onset forms of the disease with different symptoms, such as ataxia, intellectual deterioration, muscle weakness, growth retardation, fast progression, and early death $[2,8$, 11]. A few SCA17 cases, however, were recently found to have $41 \mathrm{CAG} / \mathrm{CAA}$ repeats with partial or full clinical symptoms of SCA17 [12-16]. Therefore, 41 CAG/CAA trinucleotide repeats may prove to be the critical threshold in SCA17 
[13]. Similar to other polyQ diseases, the length of the expanded polyQ repeats in SCA17 is inversely correlated with the age of onset and disease duration [2].

\section{SCA17 Pathogenesis}

\section{TBP Protein Structure}

Of all the polyQ diseases, SCA17 is caused by polyQ expansion in a well-characterized transcription factor, TBP. The TBP gene is located on chromosome $6 \mathrm{q} 27[2,3,6]$ and encodes an important general transcription initiation factor that was first identified as a component of the transcription factor IID (TFIID) complex [17]. In eukaryotes, transcription is carried out by three different RNA polymerases (I, II, and III), and TBP is recruited to RNA polymerase I, II, and III basal promoter elements, which involves either its direct binding to the TATA boxes in DNA or its protein-protein interactions in the case of TATA-less promoters [18]. The critical function of TBP in gene transcription is evidenced by the fact that TBP knockout mouse embryos do not survive beyond the blastocyst stage [19].

TBP consists of a variable $\mathrm{N}$-terminal domain and a highly conserved C-terminal domain [18]. Whereas the N-terminal domain is variable in many species, the polyQ tract is well conserved in vertebrates, although the repeat numbers vary in different species [18, 20, 21]. Many studies suggest the Cterminal core domain contains a large DNA-binding domain that interacts with DNA and that the N-terminal domain modulates the DNA-binding activity [21, 22]. More evidence has suggested that distinct subregions surrounding the polyQ tract synergistically promote the formation of the TBP-DNA complex $[22,23]$. Such a function provides a molecular basis for the idea that expanded polyQ repeats could cause conformational changes in TBP and dysregulate its transcriptional activity.

\section{TBP Accumulation and Toxicity}

It is well known that polyQ expansion causes proteins to misfold and to form neuronal intranuclear inclusions (NIIs) or aggregates, a common pathological hallmark of polyQ diseases [24]. Autopsied brains from patients with SCA17 showed TBP and NIIs accumulated in the Purkinje cell layer, cerebral cortex, neostriatum and hippocampal CA1, and subiculum $[3,25]$. Studies in a number of SCA17 animal models expressing different polyQ lengths of TBP, including transgenic and knock-in rodent models, as well as a Drosophila melanogaster model, also showed the agedependent accumulation of mutant TBP aggregates in neuronal nuclei and pronounced cerebellar degeneration, especially Purkinje cell death [26-29]. In TBP transgenic mouse models, both full-length TBP with pathologic polyQ tracts (71Q and 105Q) and truncated mutant TBP without the DNA-binding domain were found to form visible nuclear aggregates in a way reminiscent of transfected TBP in cultured cells [28, 30]. In SCA17 knock-in mice, when one copy of the mutant TBP with $105 \mathrm{Q}$ is expressed at the endogenous level, either selectively in neuronal cells or ubiquitously in all cells, mutant TBP was largely diffuse in various brain regions and formed small aggregates in the nucleus [26,31]. The different extents of TBP aggregates in transgenic mouse models that overexpress mutant TBP and TBP knock-in mice suggest that TBP aggregate formation is dependent on the expression level of TBP. Consistently, transgenic TBP mice show more severe phenotypes and earlier death than TBP knock-in mice [28, 31]. Also, the severity of phenotypes of TBP transgenic mice appear to be dependent on the length of the CAG repeat. For example, transgenic TBP-71Q mice begin to die at 11.5 weeks of age, whereas some TBP-105Q-F mice died as early as 9 weeks [28]. As expected, TBP knock-in mice that express mutant TBP with 105Q at the endogenous level start to die at the age of 6-7 months [31].

It is noteworthy that transgenic SCA17 mice expressing mutant TBP fragments lacking an intact C-terminal DNAbinding domain showed much more severe neurological symptoms, dying as early as 3 weeks [28]. This truncated mutant TBP forms aggregates more readily than full-length mutant TBP in transfected cells [28]. This phenomenon suggests that mutant TBP can induce neurotoxicity without binding to DNA and that truncated TBP is more toxic than fulllength TBP [30]. Thus, like other polyQ proteins, mutant TBP exhibits its toxicity to different extents as determined by CAG repeat length, expression level, and the context of the mutant proteins.

Accumulation of mutant TBP in the brain is age-dependent, which also supports the theory that the age-dependent decrease in clearing misfolded proteins accounts for the accumulation of polyQ proteins in aged brains [32]. Using conditional expression of mutant TBP in SCA17 knock-in mice at different ages, Yang et al. reported that older SCA17 mice experienced earlier neurological symptom onset and more severe Purkinje cell degeneration [32]. This study also revealed age-related decreases in Hsc70 and chaperone activity in mouse brains, with broad implications for the age-dependent accumulation of polyQ proteins and associated toxicity.

Expression of mutant TBP appears to lead to two important consequences. One is the formation of nuclear aggregates or inclusions (NIIs); the other is the reduction of endogenous normal TBP $[26,28,31]$. For NIIs, their roles remain to be defined, as both toxic and beneficial effects of NIIs have been reported [33-36]. Despite the undefined role of NIIs, the appearance of NIIs reflects the accumulation of mutant polyQ proteins and is correlated with disease progression. The reduction of endogenous normal TBP in SCA17 mice is interesting. 
It is quite likely that the expression level of TBP is vitally important for its critical function, such that its expression has to be tightly regulated. Mutant TBP with an expanded polyQ repeat is still able to function as a transcription factor $[30,31]$. Mutant TBP is possibly more stable than normal TBP, meaning its accumulation in aged neurons may trigger intracellular self-regulation to further reduce the level of normal TBP. As a result of decreased expression of normal TBP, loss of function of normal TBP could potentially contribute to SCA17 pathogenesis (Fig. 1).

\section{SCA17 Animal Models}

\section{Pathology in SCA17 Animal Models}

Generation of SCA17 animal models that express mutant TBP with different polyQ repeat lengths allowed for the investigation of polyQ repeat-dependent pathology (Table 1). SCA17 animal models, including mice and rats that carry expanded CAG repeats in the TBP gene, recapitulate the Purkinje cell degeneration seen in patients with SCA17 [27, 28, 37, 39]. Consistently, motor dysfunction and abnormal gait were found in these transgenic SCA17 animals. Compared to other brain regions, Purkinje cells in the cerebellum appear to be primarily affected in SCA17 rodent models.

One significant concern about using transgenic mouse models to study SCA17 pathogenesis is the ectopic overexpression of mutant TBP controlled by exogenous promoters [28], as this transgenic overexpression of polyQ proteins may lead to phenotypes that do not exist in patients, in whom only one copy of mutant TBP is expressed. A SCA17 knock-in mouse model was therefore next generated using the CreloxP technique, which allows for tissue- and time-specific expression of mutant TBP [31, 32]. In the knock-in mouse model, the mouse TBP exon 2 was replaced by human TBP exon 2 carrying 105 CAGs [26]. When mutant TBP's expression is restricted to neurons, the knock-in mouse model had similar but later-onset and milder phenotypes compared with transgenic mouse models [26]; however, when mutant TBP is ubiquitously expressed via germline transmission, mutant TBP was found to preferentially accumulate in the nuclei of

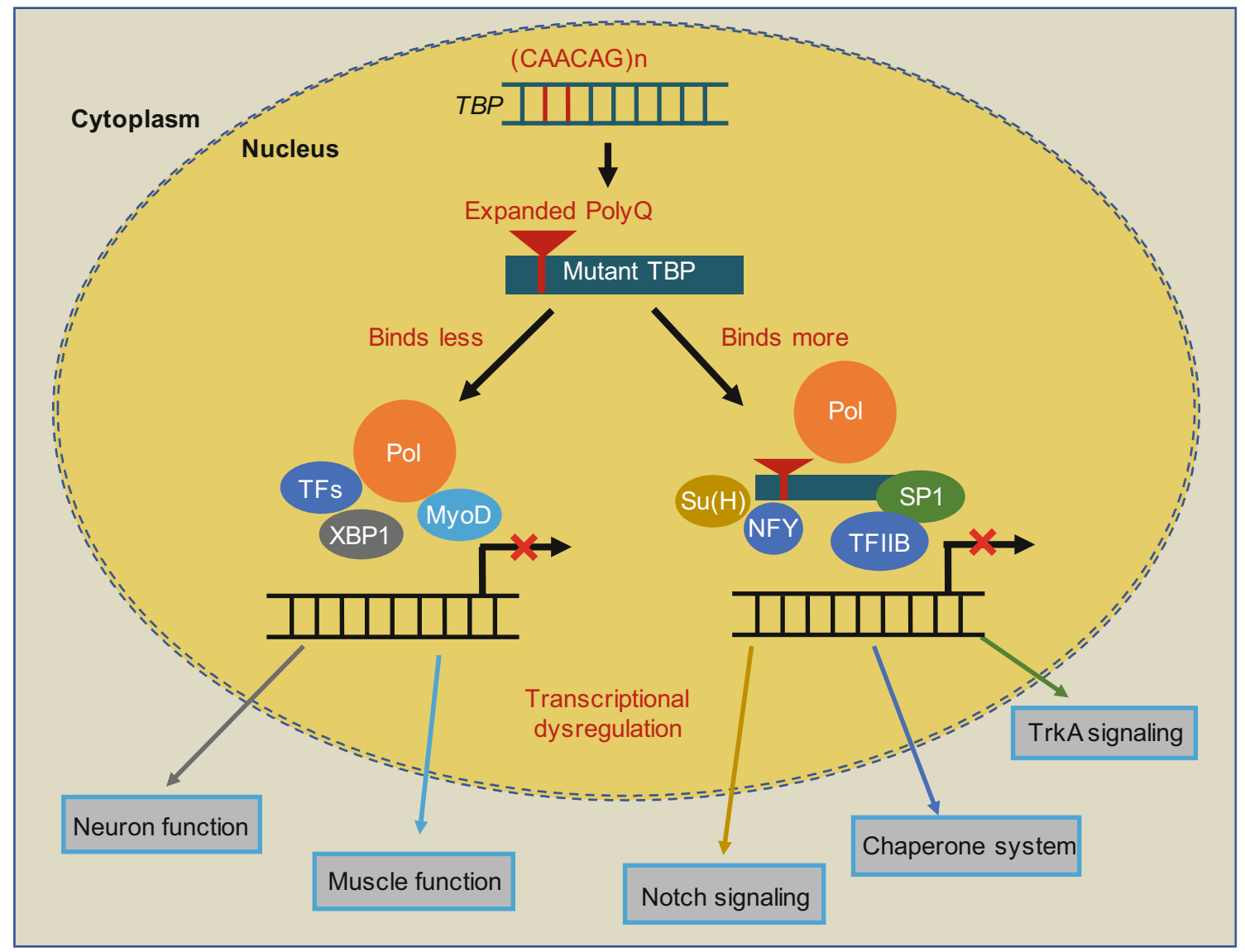

Fig. 1 The pathogenesis of SCA17. PolyQ expansion alters the association of TBP with transcription factors in the nucleus. Mutant TBP binds less to XBP1 and MyoD, affecting the formation of transcriptional complexes or their association with the DNA promoter and leading to transcriptional dysregulation. Mutant TBP can also bind more tightly to the transcription factors (NF-Y, TFIIB, RBP-J/Su $(\mathrm{H})$, SP1), thereby sequestering them and interrupting their transcriptional functions 


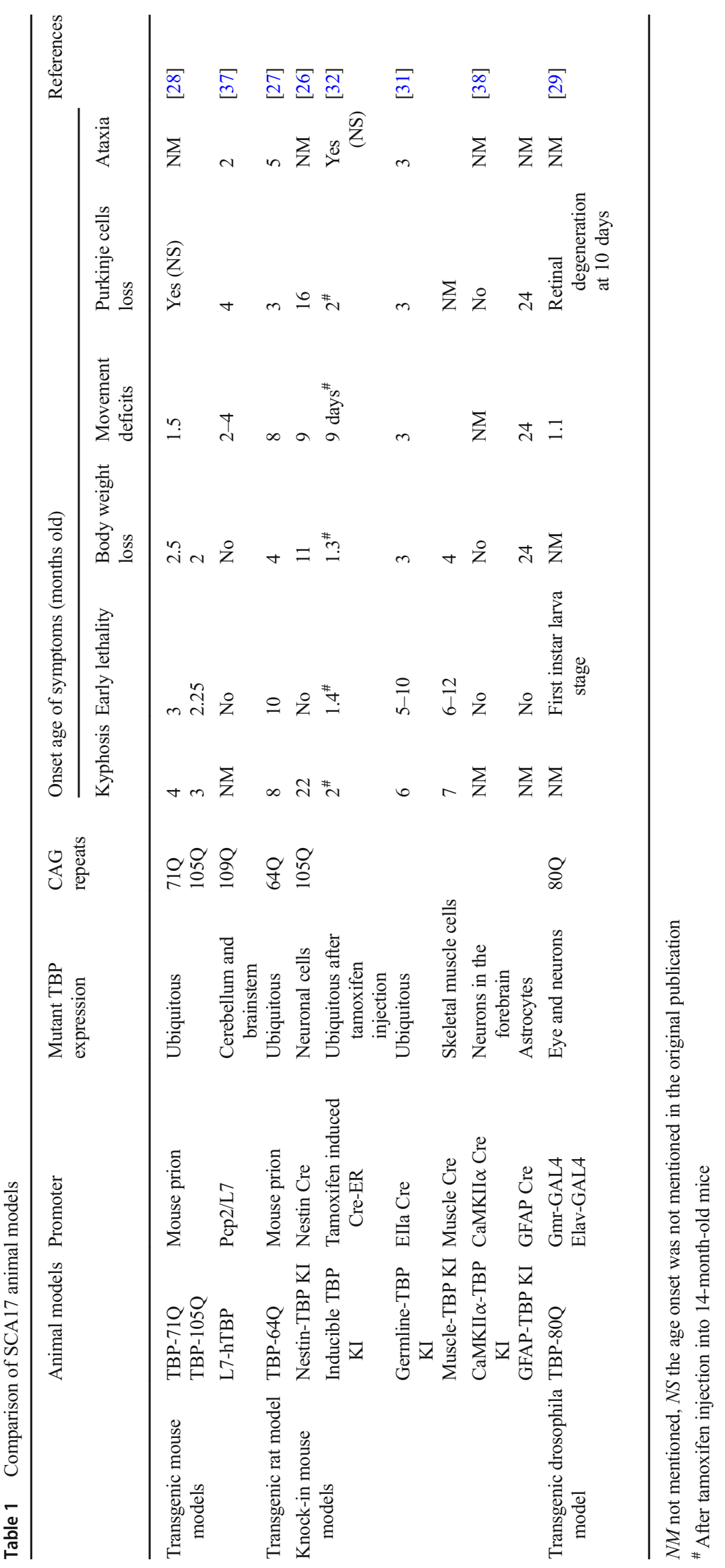


neuronal and muscle cells and yielded polyQ lengthdependent toxic effects in the brain and muscle tissues [31]. SCA17 knock-in mice that express mutant TBP with 105Q ubiquitously throughout the whole body first show striking muscle degeneration at 3 months of age, characterized by muscle fragmentation and multiple internalized or centralized nuclei, a marker of regeneration after degeneration of muscle cells [31]. Furthermore, electron microscopy examination revealed disorganized muscle fibers, Z-band disruption, sarcomere destruction, and enlarged mitochondria [31]. The more rigorous evidence for the primary effects of mutant TBP in muscles is that conditional SCA17 knock-in mice selectively expressing mutant TBP in muscle cells also showed muscle phenotypes similar to TBP-105Q KI mice. Both SCA17 knock-in mouse models display nuclear aggregates, agedependent body weight loss, movement abnormalities, and early death [31].

The striking muscle degeneration highlights the importance of polyQ protein toxicity in peripheral tissues. Mounting evidence also indicates that expanded polyQ proteins can affect muscle function in spinal and bulbar muscular atrophy (SBMA), Huntington's disease (HD), and dentatorubral-pallidoluysian atrophy (DRPLA) [40]. It should be noted that the muscle atrophy phenotype occurs in SCA17 mice that express mutant TBP with a large repeat in muscle cells. Other transgenic SCA17 mice without this peripheral phenotype could be because mutant TBP with a large polyQ repeat was not expressed in their muscle cells. There have, however, been no reports of muscle atrophy or related symptoms in adult-onset SCA17 patients who carry polyQ repeats shorter than 55 glutamines. The unique phenotype in the peripheral tissues caused by mutant TBP with 105Q may represent symptoms and pathology in juvenile patients with larger polyQ repeats, whose symptoms are distinct from those seen in adult-onset patients [8]. Despite the rarity of SCA17 cases and lack of muscle biopsy studies of juvenile SCA17 patients, two cases of juvenile-onset SCA17 patients carrying 63Q or 66Q of TBP were found to have gait abnormalities and muscle weakness at age 13 or younger, and the muscle weakness was characterized as impaired laryngeal and sphincter muscle function leading to dysphagia, dysarthria, and difficulty walking $[2,8]$. Thus, both neurodegeneration and muscle atrophy in SCA17 mice clearly demonstrate cell type- and polyQ repeat length-dependent pathology in polyQ diseases.

\section{Transcriptional Alterations}

Because TBP is a general transcription factor that plays important roles in mediating transcription by all three nuclear RNA polymerases [41], the primary mechanism of pathogenesis in SCA17 was thought to be altered transcription by the expanded polyQ repeat in TBP. Different disease models, including cells [42], Drosophila [29], and rodents [26-28, 30,
31], have provided valuable information for our understanding of SCA17 pathogenesis. In vitro studies suggest that Nterminal polyQ expansion may change the conformation of TBP to affect its DNA-binding ability [23, 30]. It is well known that the abnormal interaction of mutant polyQ proteins with various transcription factors can cause transcriptional dysregulation [43]. Expanded polyQ tracts enhanced the interaction of TBP with the general transcription factor IIB (TFIIB), and immunofluorescent results showed the colocalization of TFIIB with nuclear TBP aggregates in the cerebellum of TBP-105Q mice [28]. For example, TFIIB was sequestered to the TBP inclusions, reducing TFIIB occupancy of the Hspb1 promoter and leading to downregulation of HSPB1, a small heat shock protein. Decreased HSPB1 expression inhibited neurite outgrowth, whereas overexpression of HSPB1 or TFIIB could alleviate neuronal defects [28].

In a conditional knock-in mouse model expressing TBP105Q selectively in neuronal cells, which exhibit agedependent and progressive neurological phenotypes, mutant TBP binds more tightly to the transcription factor nuclear factor $\mathrm{Y}$ (NF-Y), a master regulator of the chaperone system [26]. NF-Y is also colocalized with nuclear TBP inclusions, suggesting that mutant TBP could sequester NF-Y to impair NF-Y-mediated expression of chaperones, such as Hsp70, Hsp25, and HspA5 [26].

In a cellular model of SCA17, TBP-105Q-expressing cells showed a greater decrease in viability and greater damaged neurite outgrowth [42]. Soluble mutant TBP bound more SP1 and inhibited its activity on the expression of TrkA, a nerve growth factor receptor. Decreased expression of TrkA was found in both the cerebellum of SCA17 mice and TBP105Q cells [42]. In a transgenic Drosophila model of SCA17 expressing mutant human TBP with $80 \mathrm{Q}$ tracts, (hTBP80Q), a transcription factor, RBP-J/Su(H), which contains $\mathrm{Q} / \mathrm{N}$-rich domains, interacted more efficiently with mutant TBP than wild-type TBP [29]. RBP-J/Su(H) participates in Notch signaling, and dysfunction of RBP-J/Su(H) contributed to hTBP80Q-induced phenotypes, including progressive retinal degeneration, late-onset locomotor impairment, and early mortality [29].

The enhanced interaction of mutant TBP with other transcription factors may sequester them from their functional locations and interrupt their normal transcriptional functions to mediate gain-of-toxic-function. The transcriptional dysregulation can also be caused by loss of function of TBP due to expanded polyQ repeats, which is well evidenced by reduced transcription of MANF in SCA17 KI mice [32]. MANF is an ER stress-inducible protein enriched in Purkinje cells, and there is an ER stress response element in the promoter region of MANF that can be recognized by transcriptional factor XBP1 during ER stress. TBP and XBP1 are reported to be present in the same transcriptional complex to mediate the expression of MANF [44] but mutant TBP has a decreased 
association with XBP1, therefore resulting in the decreased expression of MANF [32].

In terms of muscle atrophy, this phenotype seems to be caused by reduced expression of muscle-specific genes in SCA17 knock-in mice. TBP is reported to interact with MyoD, a muscle-specific transcription factor, to stabilize its binding to the DNA promoter. In SCA17 KI mice, a reduced association of mutant TBP with MyoD leads to decreased protein levels of MyoD by promoting its degradation. Meanwhile, the decreased interaction also affected the association of MyoD with the DNA promoter to reduce its transcriptional activity. These effects synergistically resulted in reduced muscle-specific gene expression, as well as muscle degeneration [31]. Strikingly, the larger polyQ repeat of TBP caused a greater reduction in the association of TBP with MyoD. Consistently, when comparing muscle histology after injecting virus expressing TBP with different polyQ repeats, the larger polyQ repeat showed more severe muscle degeneration, indicating that muscle atrophy phenotypes are dependent on the length of the polyQ repeat [31].

Taken together, because TBP binds DNA and a variety of transcription factors, polyQ expansion in TBP is likely to affect its association with DNA and transcription factors. The gene transcriptional dysregulation outcome is dependent on the polyQ repeat length in TBP, cell type-specific transcription factors associated with TBP, and DNA promoter regions that can recruit TBP and its interacting proteins. In addition to this complexity of TBP-mediated gene transcription, the cell typedependent capacity to turn over or remove misfolded TBP could also contribute to cell type-specific pathology in SCA17.

\section{Therapeutics}

Studies of the pathogenesis of SCA17 using cellular and animal models have indicated that the transcriptional dysregulation caused by mutant TBP is the key pathological event. Based on this, there are at least two therapeutic strategies for SCA17. One is to reverse the transcriptional alterations induced by mutant TBP, and the other is to reduce levels of toxic mutant TBP.

\section{Reverse Transcriptional Alterations}

Transcriptional dysregulation in SCA17 is due largely to the misfolding of mutant TBP with an expanded polyQ repeat. Prevention or reduction of this misfolding is therefore presumably beneficial to alleviate mutant TBP toxicity. The chaperone system, which can refold proteins correctly, is reported to alleviate mutant TBP-induced neuronal defects [28]. Another study found that NC009-1, an indole compound, could reduce polyQ aggregation and promote neurite outgrowth in a SCA17 cell model by upregulating HSPB1 expression [45].
Furthermore, NC009-1 could reduce polyQ aggregation in Purkinje cells and ameliorated behavioral deficits in SCA17 transgenic mice [45]. There are also some other drugs that can alleviate mutant TBP-induced neurotoxicity by increasing the levels of NFYA or Hsp70 [46, 47]. Increasing the expression of specific molecular chaperones or quality control ubiquitin ligases are expected to help protein folding and have been found to alleviate disease phenotypes in a variety of cellular and animal models of polyQ diseases [45-50].

Among many downstream molecules associated with altered gene transcription in SCA17 cellular and mouse models, MANF is a promising one that can serve as a therapeutic target. In SCA17 KI mice, expression of MANF is downregulated, owing to the decreased transcriptional activity of XBP1 caused by mutant TBP [32]. MANF functions extracellularly as a neuronal protective factor through PKC signaling and also acts intracellularly as an ER stress-inducible protein $[32,51]$. Overexpression of MANF can successfully ameliorate mutant TBP-mediated Purkinje cell degeneration and other pathological phenotypes [32]. Thus, drugs and chemicals capable of upregulating MANF levels can be used to test their therapeutic effects. Indeed, a large-scale screening study identified an FDA-approved chemical, piperine, which could increase the expression of MANF and reduce cellular ER stress. Treating SCA17 KI mice with piperine significantly improved both behavioral performances and neuropathology [52]. This protection is also consistent with the anti-inflammatory effect of piperine [38, 53, 54]. Uncovering the therapeutic effect of piperine opens up a new avenue to search for other drugs with similar structure and function that can more efficiently treat SCA17 or other diseases that are also caused by misfolded polyQ proteins. Drugs that have been used for treating SCA17 cellular and mouse models are listed in Table 2.

\section{Reducing Mutant TBP}

Because mutant TBP leads to complex toxicity by affecting multiple gene transcriptions, reducing the level of toxic mutant TBP should be a more effective therapeutic strategy. Although currently such a strategy has not been used in SCA17 models, it has been tested in other polyQ disease models [57]. Using CRISPR-Cas9, antisense oligonucleotides (ASOs), small interfering RNAs (siRNAs), short hairpin RNAs (shRNAs), or artificial microRNAs (miRNAs) to knock down RNA of disease genes has shown promising therapeutic efficacy in animal models of polyQ diseases, such as HD [58, 59], SCA3 [60], SCA6 [61], and SCA7 [62]. Compared to therapeutic strategies that require continuous administration of therapeutic agents into the brain, CRISPRCas9-mediated gene targeting appears to be more efficient, as this gene editing method can permanently deplete the expression of mutant proteins. Stereotaxic injection of viral vectors expressing CRSIPR-Cas9 in the mouse brain has been used to 
Table 2 Candidate drugs for treatment of SCA17

\begin{tabular}{|c|c|c|c|c|}
\hline Drugs & Ingredients & Targeted molecules & Pre-clinical studies & References \\
\hline Trehalose & Disaccharide & $\mathrm{NM}$ & $\begin{array}{l}\text { Reduced aggregation and ameliorated behavioral performances } \\
\text { in SCA17 transgenic mice }\end{array}$ & {$[55]$} \\
\hline EGb 761 & Ginkgo biloba extract & NM & $\begin{array}{l}\text { Reduced aggregation in the TBP } / 79 Q \text { cells model; } \\
\text { Ameliorated motor function in SCA17 transgenic mice }\end{array}$ & {$[56]$} \\
\hline G-CSF & Glycoprotein & $\begin{array}{l}\text { Hsp70 } \\
\text { Beclin-1 LC3-II }\end{array}$ & $\begin{array}{l}\text { Improved motor coordination; } \\
\text { Reduced cell loss and aggregation in SCA17 transgenic mice }\end{array}$ & {$[46]$} \\
\hline Piperine & Alkaloid & MANF & $\begin{array}{l}\text { Reduced ER stress in TBP } / 105 \mathrm{Q} \text { cell model; } \\
\text { Improved Purkinje cell survival } \\
\text { and behavioral performances in SCA17 knock-in mice }\end{array}$ & {$[52]$} \\
\hline NC009-1 & Indole compound & HSPB1 & $\begin{array}{l}\text { Promoted neurite outgrowth in SCA17 cell model; } \\
\text { Reduced aggregation and ameliorated behavioral deficits in } \\
\text { SCA17 transgenic mice }\end{array}$ & {$[45]$} \\
\hline SG-Tang & Formulated Chinese medicine & $\begin{array}{l}\text { NFYA } \\
\text { PGC-1a } \\
\text { NRF2 }\end{array}$ & $\begin{array}{l}\text { Reduced aggregation and promoted neurite outgrowth in } \\
\text { SCA17 cell model; } \\
\text { Ameliorated behavioral deficits and reduced aggregation } \\
\text { in SCA17 transgenic mice }\end{array}$ & {$[47]$} \\
\hline
\end{tabular}

$N M$ not mentioned

treat HD mice and yielded promising therapeutic effects [58]; however, there are still some challenges with gene editing approaches, including brain delivery efficiency and off-target effects [63]. In addition, because TBP is very important for transcription and its expression is tightly regulated, mutant allele-specific silencing of TBP is highly recommended when treating SCA17. Given that TBP is a well-characterized transcription factor, SCA17 would provide us with an ideal model to test whether selective removal of the polyQ repeat in TBP via CRISPR-Cas9 would beneficially eliminate polyQ toxicity without significantly affecting the important function of TBP.

Because of the striking muscle pathology in SCA17 knockin mice, they also provide us with an excellent model to test therapeutics on the peripheral pathology caused by mutant polyQ proteins. Targeting the mutant polyQ protein itself as the proximal mediator of disease has attracted much recent attention. In the SBMA disease mouse model, gene silencing techniques, such as antisense oligonucleotides and the CreloxP system, were applied to selectively suppress the expression of mutant polyQ-AR in skeletal muscle [64, 65]. Because CRISPR-Cas9 expression in muscle cells is readily achieved by viral transduction, CRISPR-Cas9 would make a very useful tool to assess whether removing the CAG repeat or part of the polyQ disease gene in SCA17 mice can successfully achieve a therapeutic goal.

\section{Conclusions}

Although SCA17 is a rare disease, mouse models of it would be highly valuable for investigating the pathogenesis of the polyQ diseases. This is because the function of TBP is well characterized, enabling the study of how polyQ repeat expansion affects important protein function. In particular, the transcriptional function of TBP allows us to investigate how polyQ expansion affects gene transcription, an important pathogenic mechanism that also underlies other polyQ diseases. The repeat length-dependent phenotypes, as well as specific pathology in the brain and muscle of SCA17 mice, provide important pathological targets for developing effective therapeutic strategies. Some promising therapeutic approaches, such as inhibiting protein misfolding by drugs and reducing the expression of the disease genes by antisense oligonucleotides or CRISPR-Cas9, can also be applied to animal models of SCA17. Success in treating SCA17 animal models would have broad implications for developing effective treatments for other polyQ diseases.

Required Author Forms Disclosure forms provided by the authors are available with the online version of this article.

Disclosures The authors have no financial disclosures to report related to this article.

\section{Compliance with Ethical Standards}

Conflict of Interest The authors declare that they have no conflicts of interest.

\section{References}

1. Orr HT and Zoghbi HY. Trinucleotide repeat disorders. Annu Rev Neurosci 2007;30:575-621.

2. Koide R, Kobayashi S, Shimohata T, et al. A neurological disease caused by an expanded CAG trinucleotide repeat in the TATAbinding protein gene: a new polyglutamine disease? Hum Mol Genet 1999:8:2047-2053.

3. Nakamura K, Jeong SY, Uchihara T, et al. SCA17, a novel autosomal dominant cerebellar ataxia caused by an expanded 
polyglutamine in TATA-binding protein. Hum Mol Genet 2001;10: 1441-1448.

4. Bech S, Petersen T, Norremolle A, et al. Huntington's disease-like and ataxia syndromes: identification of a family with a de novo SCA17/TBP mutation. Parkinsonism Relat Disord 2010;16:12-15.

5. Shatunov A, Fridman EA, Pagan FI, et al. Small de novo duplication in the repeat region of the TATA-box-binding protein gene manifest with a phenotype similar to variant Creutzfeldt-Jakob disease. Clin Genet 2004;66:496-501.

6. Fujigasaki H, Martin JJ, De Deyn PP, et al. CAG repeat expansion in the TATA box-binding protein gene causes autosomal dominant cerebellar ataxia. Brain 2001;124:1939-1947.

7. Gao R, Matsuura T, Coolbaugh M, et al. Instability of expanded CAG/CAA repeats in spinocerebellar ataxia type 17. Eur J Hum Genet 2008; 16:215-222.

8. Maltecca F, Filla A, Castaldo I, et al. Intergenerational instability and marked anticipation in SCA-17. Neurology 2003;61:14411443.

9. Bruni AC, Takahashi-Fujigasaki J, Maltecca F, et al. Behavioral disorder, dementia, ataxia, and rigidity in a large family with TATA box-binding protein mutation. Arch Neurol 2004;61:1314 1320.

10. Rolfs A, Koeppen AH, Bauer I, et al. Clinical features and neuropathology of autosomal dominant spinocerebellar ataxia (SCA17). Ann Neurol 2003;54:367-375.

11. Rubinsztein DC, Leggo J, Crow TJ, et al. Analysis of polyglutamine-coding repeats in the TATA-binding protein in different human populations and in patients with schizophrenia and bipolar affective disorder. Am J Med Genet 1996;67:495-498.

12. Park H, Jeon BS, Shin JH, and Park SH. A patient with 41 CAG repeats in SCA17 presenting with parkinsonism and chorea. Parkinsonism Relat Disord 2016;22:106-107.

13. Origone P, Gotta F, Lamp M, et al. Spinocerebellar ataxia 17: full phenotype in a $41 \mathrm{CAG} / \mathrm{CAA}$ repeats carrier. Cerebellum Ataxias 2018;5:7.

14. Nanda A, Jackson SA, Schwankhaus JD, and Metzer WS. Case of spinocerebellar ataxia type 17 (SCA17) associated with only 41 repeats of the TATA-binding protein (TBP) gene. Mov Disord 2007;22:436

15. Alibardi A, Squitieri F, Fattapposta F, et al. Psychiatric onset and late chorea in a patient with $41 \mathrm{CAG}$ repeats in the TATA-binding protein gene. Parkinsonism Relat Disord 2014;20:678-679.

16. Doherty KM, Warner TT, and Lees AJ. Late onset ataxia: MSA-C or SCA 17? A gene penetrance dilemma. Mov Disord 2014;29:3638 .

17. Burley SK and Roeder RG. Biochemistry and structural biology of transcription factor IID (TFIID). Annu Rev Biochem 1996;65:769799.

18. Hernandez N. TBP, a universal eukaryotic transcription factor? Genes Dev 1993;7:1291-1308.

19. Martianov I, Viville S, and Davidson I. RNA polymerase II transcription in murine cells lacking the TATA binding protein. Science 2002;298:1036-1039.

20. Shimada M, Ohbayashi T, Ishida M, et al. Analysis of the chicken TBP-like protein(tlp) gene: evidence for a striking conservation of vertebrate TLPs and for a close relationship between vertebrate tbp and tlp genes. Nucleic Acids Res 1999;27:3146-3152.

21. Horikoshi M, Yamamoto T, Ohkuma Y, Weil PA, and Roeder RG. Analysis of structure-function relationships of yeast TATA box binding factor TFIID. Cell 1990;61:1171-1178.

22. Davidson I. The genetics of TBP and TBP-related factors. Trends Biochem Sci 2003;28:391-398.

23. Zhao $X$ and Herr W. A regulated two-step mechanism of TBP binding to DNA: a solvent-exposed surface of TBP inhibits TATA box recognition. Cell 2002;108:615-627.
24. Yamada M, Tsuji S, and Takahashi H. Pathology of CAG repeat diseases. Neuropathology 2000;20:319-325.

25. Toyoshima Y and Takahashi H. Spinocerebellar Ataxia Type 17 (SCA17). Adv Exp Med Biol 2018;1049:219-231.

26. Huang S, Ling JJ, Yang S, Li XJ, and Li S. Neuronal expression of TATA box-binding protein containing expanded polyglutamine in knock-in mice reduces chaperone protein response by impairing the function of nuclear factor-Y transcription factor. Brain 2011;134: 1943-1958.

27. Kelp A, Koeppen AH, Petrasch-Parwez E, et al. A novel transgenic rat model for spinocerebellar ataxia type 17 recapitulates neuropathological changes and supplies in vivo imaging biomarkers. J Neurosci 2013;33:9068-9081.

28. Friedman MJ, Shah AG, Fang ZH, et al. Polyglutamine domain modulates the TBP-TFIIB interaction: implications for its normal function and neurodegeneration. Nat Neurosci 2007;10:15191528.

29. Ren J, Jegga AG, Zhang M, et al. A Drosophila model of the neurodegenerative disease SCA17 reveals a role of $\mathrm{RBP}-\mathrm{J} / \mathrm{Su}(\mathrm{H})$ in modulating the pathological outcome. Hum Mol Genet 2011;20: 3424-3436.

30. Friedman MJ, Wang CE, Li XJ, and Li S. Polyglutamine expansion reduces the association of TATA-binding protein with DNA and induces DNA binding-independent neurotoxicity. J Biol Chem 2008;283:8283-8290.

31. Huang S, Yang S, Guo J, et al. Large Polyglutamine Repeats Cause Muscle Degeneration in SCA17 Mice. Cell Rep 2015;13:196-208.

32. Yang S, Huang S, Gaertig MA, Li XJ, and Li S. Age-dependent decrease in chaperone activity impairs MANF expression, leading to Purkinje cell degeneration in inducible SCA17 mice. Neuron 2014;81:349-365.

33. Arrasate M, Mitra S, Schweitzer ES, Segal MR, and Finkbeiner S Inclusion body formation reduces levels of mutant huntingtin and the risk of neuronal death. Nature 2004;431:805-810.

34. Bowman AB, Lam YC, Jafar-Nejad P, et al. Duplication of Atxn11 suppresses SCA1 neuropathology by decreasing incorporation of polyglutamine-expanded ataxin-1 into native complexes. Nat Genet 2007;39:373-379.

35. Saudou F, Finkbeiner S, Devys D, and Greenberg ME. Huntingtin acts in the nucleus to induce apoptosis but death does not correlate with the formation of intranuclear inclusions. Cell 1998;95:55-66.

36. Klement IA, Skinner PJ, Kaytor MD, et al. Ataxin-1 nuclear localization and aggregation: role in polyglutamine-induced disease in SCA1 transgenic mice. Cell 1998;95:41-53.

37. Chang YC, Lin CY, Hsu CM, et al. Neuroprotective effects of granulocyte-colony stimulating factor in a novel transgenic mouse model of SCA17. J Neurochem 2011;118:288-303.

38. Yang Y, Yang S, Guo J, et al. Synergistic Toxicity of Polyglutamine-Expanded TATA-Binding Protein in Glia and Neuronal Cells: Therapeutic Implications for Spinocerebellar Ataxia 17. J Neurosci 2017;37:9101-9115.

39. Portal E, Riess O, and Nguyen HP. Automated home cage assessment shows behavioral changes in a transgenic mouse model of spinocerebellar ataxia type 17. Behav Brain Res 2013;250:157165.

40. Huang S, Zhu S, Li XJ, and Li S. The Expanding Clinical Universe of Polyglutamine Disease. Neuroscientist 2019: 1073858418822993.

41. Nikolov DB and Burley SK. 2.1 A resolution refined structure of a TATA box-binding protein (TBP). Nat Struct Biol 1994;1:621-637.

42. Shah AG, Friedman MJ, Huang S, et al. Transcriptional dysregulation of TrkA associates with neurodegeneration in spinocerebellar ataxia type 17. Hum Mol Genet 2009;18:4141-4152.

43. Riley BE and Orr HT. Polyglutamine neurodegenerative diseases and regulation of transcription: assembling the puzzle. Genes Dev 2006;20:2183-2192. 
44. Hetz C. The unfolded protein response: controlling cell fate decisions under ER stress and beyond. Nat Rev Mol Cell Biol 2012;13: 89-102.

45. Chen CM, Chen WL, Hung CT, et al. The indole compound NC009-1 inhibits aggregation and promotes neurite outgrowth through enhancement of HSPB1 in SCA17 cells and ameliorates the behavioral deficits in SCA17 mice. Neurotoxicology 2018;67: 259-269.

46. Chang YC, Lin CW, Hsu CM, et al. Targeting the prodromal stage of spinocerebellar ataxia type 17 mice: G-CSF in the prevention of motor deficits via upregulating chaperone and autophagy levels. Brain Res 2016;1639:132-148.

47. Chen CM, Chen WL, Hung CT, et al. Shaoyao Gancao Tang (SGTang), a formulated Chinese medicine, reduces aggregation and exerts neuroprotection in spinocerebellar ataxia type 17 (SCA17) cell and mouse models. Aging (Albany NY) 2019;11:986-1007.

48. Ding Y, Adachi H, Katsuno M, et al. BIIB021, a synthetic Hsp90 inhibitor, induces mutant ataxin-1 degradation through the activation of heat shock factor 1. Neuroscience 2016;327:20-31.

49. Cushman-Nick M, Bonini NM, and Shorter J. Hsp104 suppresses polyglutamine-induced degeneration post onset in a drosophila MJD/SCA3 model. PLoS Genet 2013;9:e1003781.

50. Williams AJ, Knutson TM, Colomer Gould VF, and Paulson HL. In vivo suppression of polyglutamine neurotoxicity by $\mathrm{C}$-terminus of Hsp70-interacting protein (CHIP) supports an aggregation model of pathogenesis. Neurobiol Dis 2009;33:342-353.

51. Voutilainen MH, Back S, Porsti E, et al. Mesencephalic astrocytederived neurotrophic factor is neurorestorative in rat model of Parkinson's disease. J Neurosci 2009;29:9651-9659.

52. Guo J, Cui Y, Liu Q, et al. Piperine ameliorates SCA17 neuropathology by reducing ER stress. Mol Neurodegener 2018;13:4.

53. Dong Y, Huihui Z, and Li C. Piperine inhibit inflammation, alveolar bone loss and collagen fibers breakdown in a rat periodontitis model. J Periodontal Res 2015;50:758-765.

54. Bang JS, Oh DH, Choi HM, et al. Anti-inflammatory and antiarthritic effects of piperine in human interleukin 1beta-stimulated fibroblast-like synoviocytes and in rat arthritis models. Arthritis Res Ther 2009; 11:R49.

55. Chen ZZ, Wang CM, Lee GC, et al. Trehalose attenuates the gait ataxia and gliosis of spinocerebellar ataxia type 17 mice. Neurochem Res 2015;40:800-810.
56. Huang DS, Lin HY, Lee-Chen GJ, et al. Treatment with a Ginkgo biloba extract, EGb 761, inhibits excitotoxicity in an animal model of spinocerebellar ataxia type 17. Drug Des Devel Ther 2016;10: 723-731.

57. Keiser MS, Kordasiewicz HB, and McBride JL. Gene suppression strategies for dominantly inherited neurodegenerative diseases: lessons from Huntington's disease and spinocerebellar ataxia. Hum Mol Genet 2016;25:R53-64.

58. Yang S, Chang R, Yang H, et al. CRISPR/Cas9-mediated gene editing ameliorates neurotoxicity in mouse model of Huntington's disease. J Clin Invest 2017;127:2719-2724.

59. Tabrizi S, Leavitt B, Kordasiewicz H, et al. Effects of IONISHTTRx in Patients with Early Huntington's Disease, Results of the First HTT-Lowering Drug Trial (CT.002). Neurology 2018;90:CT.002.

60. Rodriguez-Lebron E, Costa Mdo C, Luna-Cancalon K, et al. Silencing mutant ATXN3 expression resolves molecular phenotypes in SCA3 transgenic mice. Mol Ther 2013;21:1909-1918.

61. Miyazaki Y, Du X, Muramatsu S, and Gomez CM. An miRNAmediated therapy for SCA6 blocks IRES-driven translation of the CACNA1A second cistron. Sci Transl Med 2016;8:347-394.

62. Ramachandran PS, Boudreau RL, Schaefer KA, La Spada AR, and Davidson BL. Nonallele specific silencing of ataxin-7 improves disease phenotypes in a mouse model of SCA7. Mol Ther 2014;22:1635-1642.

63. Maxwell KL. The Anti-CRISPR Story: A Battle for Survival. Mol Cell 2017;68:8-14.

64. Cortes CJ, Ling SC, Guo LT, et al. Muscle expression of mutant androgen receptor accounts for systemic and motor neuron disease phenotypes in spinal and bulbar muscular atrophy. Neuron 2014;82: 295-307.

65. Lieberman AP, Yu Z, Murray S, et al. Peripheral androgen receptor gene suppression rescues disease in mouse models of spinal and bulbar muscular atrophy. Cell Rep 2014;7:774-784.

Publisher's Note Springer Nature remains neutral with regard to jurisdictional claims in published maps and institutional affiliations. 